\title{
On the branch and cut method for multidimentional mixed integer Knapsack problem
}

\author{
Mostafa Khorramizadeh*, Zahra Rakhshandehroo \\ Department of Mathematical Sciences, Shiraz University of Technology, Shiraz 71555-313, Iran \\ *Corresponding author E-mail: m.khorrami@sutech.ac.ir
}

\begin{abstract}
Copyright (C)2014 Mostafa Khorramizadeh and Zahra Rakhshandehroo. This is an open access article distributed under the Creative Commons Attribution License, which permits unrestricted use, distribution, and reproduction in any medium, provided the original work is properly cited.
\end{abstract}

\begin{abstract}
In this paper, we examine the effect of the feasibility pump (FP) method on the branch and cut method for solving the multidimentional mixed integer knapsack problem. The feasibility pump is a heuristic method, trying to compute a feasible solution for mixed integer programming problems. Moreover, we consider two efficient strategies for using the feasibility pump in a branch and cut method and present some tables of numerical results, concerning the application and comparison of using these strategies in the branch and cut method for solving the multidimentional mixed integer knapsack problem. The numerical results indicate that for the majority of the test problems, by using the FP or the imroved version of the FP we can improve the efficiency of the branch and cut method for solving the multidimentional mixed integer knapsack problem.
\end{abstract}

Keywords: Feasibility pump method, Branch and cut method, multidimentional mixed integer knapsack problem.

\section{Introduction}

Given a collection of items having both a weight and a usefulness, the knapsack problem is to fill a bag whose capacity is constrained while maximizing the sum of the usefulness of the items contained in the bag. In the mixed integer knapsack problem the number of some items are allowed to be continuous while the number of the remaining variables must be integer. The mixed integer knapsack problem belongs to the class of NP-hard problems [5]. The original version of the knapsack problem has one constraint relating to the capacity of the bag, while in the multidimentional knapsack problem we have several constraints of this type. This problem has many applications in the mathematical sciences and engineering [5, 7]. On efficient algorithm for solving the multidimentional mixed integer knapsack problem is the branch and cut method [1,6]. Recently, Bertacco et al. [3] presented an efficient heuristic algorithm, called the feasibiltiy pump, for finding a feasible solution of mixed integer programming problems and showed that their heuristic algorithm is able to improve the efficiency of the branch and cut method. Then, Boland et al. [2] introduced an improvement of the feasibility pump method. One important issue is the study of the effect of the feasibity pump method to a branch and cut method when applied to solve the multidimentional mixed integer knapsack problem.

In this paper we first describe original version of the FP method [3] for computing a feasible solution of the mixed integer programming problems. Then, we explain the improved version of the FP, presented in [2]. The original version of the FP tries to find the feasible solutions without taking objective function into account, while the improved version usually finds solutions with better objective value, but is more likely to fail to find a feasible 
solution [2]. Then, we apply three branch and cut methods to a set of multidimentional mixed integer knapsack standard test problems, taken from [1]. The first branch and cut method uses the original version of the FP, while the second branch and cut method uses the improved version of the FP. In the third branch and cut method we do not use the FP method. Finally, we present some numerical results to examine the effect of using the original version of FP and the improved version of FP on the implemented branch and cut methods for solving multidimentional mixed integer knapsack problem instances. We apply the implemented algorithms on 90 standard rest probelms. The numerical results show that for the majority of the test problem instances, by using the FP or the imroved version of the FP we can improve the efficiency of the branch and cut method for solving the multidimentional mixed integer knapsack problem.

In section 2, we describe the original and improved versions of the FP. In section 3, we study the steps of the branch and cut method. In section 4, we consider the multidimentional mixed integer knapsack problem. Finally, section 5 is devoted to the numerical results.

\section{Feasibility pump method}

In this section we explain the steps of the original and improved versions of the FP. Consider the following mixed integer programming problem:

$\min \left\{c^{T} x \mid A x \leq b, l \leq x \leq u, x_{j} \in Z \forall j \in I\right\} \quad(M I P)$

In the first step of the $\mathrm{FP}$ we round the solution $x^{*}$ of the LP relaxation

$\min \left\{c^{T} x \mid ; A x \leq b, l \leq x \leq u,\right\} \quad(L P)$

to an integer vector $\tilde{x}=[x]^{I}$. For every $S \subset N$ let $[x]^{S}$ be so that

$\left[x_{j}\right]^{S}= \begin{cases}{\left[x_{j}+0 / 5\right]} & \text { if } j \in S \\ x_{j} & \text { ifj } \notin S .\end{cases}$

In case $\tilde{x}$ is not feasible, we try to compute a vector in the set of feasible solutions of the LP relaxatuon, that minimizes $\Delta(x, \tilde{x})=\sum_{j \in I}\left|x_{j}-\tilde{x}_{j}\right|$. The FP replaces $x^{*}$ with the new computed vector and proceed. Moreover, FP terminates if $x^{*}=\tilde{x}$ or a predifined iteration limit is reached. Note that the closest point in the feasible set of the LP relaxation to $\tilde{x}$ is computed by solving the followhng LP problem:

$$
\begin{array}{cc}
\min & \Delta(x, \tilde{x})=\sum_{\substack{j \in I \\
\tilde{x}_{j}=l_{j}}}\left(x_{j}-l_{j}\right)+\sum_{\substack{j \in I \\
\tilde{x}_{j}=u_{j}}}\left(u_{j}-x_{j}\right)+\sum_{\substack{j \in I \\
l_{j}<\tilde{x}_{j}<u_{j}}} d_{j}, \\
\text { s.t. } & A x \leq b, d \geq x-\tilde{x}, d \geq \tilde{x}-x, l \leq x \leq u,
\end{array}
$$

where, the variables $d_{j}$ model the nonlinear term $d_{j}=\left|x_{j}-\tilde{x}_{j}\right|$ for integer variables $x_{j}$ that are not equal to one of their bounds in the rounded solution $\tilde{x}$. During the algorithm the same sequence of integer and LP-feasible points can be visited over and over again. To overcome this difficulty, each time an integer point $\tilde{x}$ is generated that was already visited in a prior iteration, we perform a restart. In a restart a random perturbation step is executed, which shifts some of the variables randomly up or down and installs this perturbed vector as new integer point $\tilde{x}$ to continue the search. The second isue accurs if for a larg number of iterations, there is no considerable improvment in the fractional measure

$f\left(x^{*}\right):=\sum_{j \in I}\left(f\left(x_{j}^{*}\right)\right) \quad, \quad f\left(x_{j}^{*}\right):=\left|x_{j}^{*}-\left[x_{j}^{*}+0 / 5\right]\right|$.

To deal with the second isue we perform a restart if in a certain number of iterations no larg number of improvment is observed in fractional measure. For fractional considrations, the original vergen of the FP consists of three stages. In the first stage we relax the integrality conditions on the general integer variables and perform a certain number of iterations (called a pumping round) just on the binary variables $B \subset I$. If the performance of the first stage dose not give us the feasible solution, we apply the second stage. We start the second stage from the initial point $\tilde{x}$ which is the closest point to the set of feasible solutions of the LP, that was visited during the first stage. Then, we execute a certain number of iterations on all integer variables. If no feasible solution is found by performing the 
second stage, we enter the third stage. In the third stage we use a point $\tilde{x}$ from the second stage closest to the set of feasible solutions of LP and solve the MIP:

$\min \left\{\Delta(x, \tilde{x}) \mid A x \leq b, l \leq x \leq u, x_{j} \in Z \forall j \in I\right\}$

The third stage stops if the first feasible solution is found or if a a certain iteration limit is reached.

Next we describe the improved version of the FP. The main idea of the improved FP is to find a feasible solution $x$ of the problem for wich the objective value $c^{T} x$ is as small as possible. In [2] Boland et al. considerd this issue by gradually reducing the influence of the original objective function $c^{T} x$ and increasing the weight of the artificial objective function of the FP $\Delta$. Indeed assume that $c \neq 0$. In the improved FP the distance function $\Delta(., \tilde{x})$ is replaced with a convex combination of $\Delta$ and $c$ :

$\Delta_{\alpha}(x, \tilde{x})=(1-\alpha) \Delta(x, \tilde{x})+\alpha \frac{\|\Delta\|}{\|c\|} c^{T} x, \quad \alpha \in[0,1]$,

where $\|$.$\| is the Euclidean norm of a vector, and \Delta$ is the objective function vector of the original FP. In the first stage we have $\|\Delta\|=\sqrt{|B|}$ and in the second stage we have $\|\Delta\|=\sqrt{|I|}$. In pumping rounds $\alpha$ is decreased geometricly, i.e. $\alpha_{i+1}=\phi \alpha_{i}$ and $\alpha_{0} \in[0,1]$ where $\phi<1$ and $\alpha_{i}$ denotes the value of $\alpha_{i}$ in the $i$ th iteration. In the improved version of the FP, we avoid cycling as follows. We remember paires $\left(\tilde{x}, \alpha_{i}\right)$ and in the $i$ th iteration, we perform a restart if $\tilde{x}$ was visited at itration $i^{\prime}<i$ with $\alpha_{i^{\prime}}-\alpha_{i} \leq \delta_{\alpha}$ and $\delta_{\alpha} \in[0,1]$, see [2] for details.

\section{Branch and cut method}

In this section we present an overview of the branch and cut method for solving the MIP [7, 4]. The branch and cut method is a generization of the branch and bound method and consists of three major parts. The first part is called the enumerative part. In the enumerative part we perform the initialazation, bounding, fixing and setting of the variables, bounding, selection and fathoming. The initialization is concerned with operations such as reading in the problem data, setting of the parametes of the algorithm and etc. In the bounding part we perform some operations relating to the update of the best solution fund so far. By fixing and setting of variables we mean the assignment of a constant value to some variables of the problem, in the remainder of the branch and cut method. In the selection part we choose an active node of the branch and cut tree for processing. The selection of the next node can be perfomed according to the depth first search, the breath first search and the best-node first search criteria. In the fathoming part we stop processing a node further if the optimal value of the LP ralaxation of this node is greater than or equal to the best optimal value found so far or if the LP relaxation of the node is infeasible or the optimal solution of the LP relaxation of the node is feasible for the MIP. Another criteria for fathoming is called fathomming by contradiction. Fathomming by contradiction occures if we observe that some fixed variables must be fixed or set to another value. Finally a node is fathomed if the tailing off happenes, i.e. for certain number of successive iterations the optimal value of the LP relaxation of the corresponding nodes does not improved considerably.

The second part of the branch and cut algorithm is concened with the computation of lower bound. This part consists of the initialization of the new node, solving of the coressponding LP, separation and elimination. After initialization of the new node and solving the corresponding LP, in the separation part, we generate a valid inequality that cuts off the fractional optimal solution of the LP. In the elimination part we eliminate some valid inequaliteis that are not effective.

In the third major part of the branch and cut method, we compute an upper bound. In this part we try to find a feasible solution for the MIP and improve quality of the best solution found so far, in the every node of the branch and cut tree. The main idea of this part is that if we can improve the quality of the best solution so far, then we can decrease the width of the branch and cut tree and save a lot of computational cost. This strategy is prooved to be effective in the practice $[3,2]$. One of the most efficient heuristic methods for finding a feasible solution of MIP, relating to this part is the $\mathrm{FP}$ method.

\section{Mixed integer knapsack model}

In this section we briefly describe the mixed integer programming formulation of the multidimentional mixed integer knapsack problem [1]. In the following let $I$ and $C$ be the set of indices of integer and continuous variables, respectively. For every $i \in I$, let $x_{i}$ and $c_{i}$ denote the number and the usefulness of the $i$ th integer item, respectively. 
For every $j \in C$, let $w_{j}$ and $d_{j}$ denote the number and the usefulness of the $j$ th continuous item, respectively. Then, the mixed integer programming formulation of the multidimentional mixed integer knapsack problem is as follows:

$$
\begin{array}{ccc}
\max & \sum_{i \in I} c_{i} x_{i}+\sum_{j \in J} d_{j} w_{j} & \\
\text { s.t. } & \sum_{i \in I} a_{i r} x_{i}+\sum_{j \in J} g_{j r} w_{j} \leq b_{r}, & r \in R, \\
x_{i} \leq u_{i}, \quad w_{j} \leq v_{j}, & i \in I, j \in C, \\
x_{i} \geq 0, \quad w_{j} \geq 0, & i \in I, j \in C .
\end{array}
$$

where, $b_{r}$ is the capacity correspoding to the $r$ th constraint and $a_{i r}$ and $g_{j r}$ are the space occupied in the $r$ th constraint by the $i$ th integer variable and the $j$ th continuous variable, respectively. $u_{i}$ and $v_{j}$ denote the upper bound on the $i$ th integer variable and the $j$ th continuous variable, respectively. Moreover, no sign restriction is imposed on $b_{r}, a_{i r}$ and $g_{j r}$ and they can take negative values.

\section{Numerical results}

In this section we describe our computational experiments. For the implementation we used a camputer with a a 2.53GHz Corei3/Windows7 with 2GB RAM using the CPLEX Version 12.6 with default settings. The 90 problem instances are taken from [1]. Table 1 is concerned with the properties of the generated test problems. In this table $n p$ denotes the problem number, $|I|,|C|$ and $|R|$ denote the number of integer variabes, the number of continuous variables and the number of constraints of the generated test problem, respectively. Tables 2 and 3 are devoted to the comparison of the computing time and the number of nodes of the branch and cut tree for solving the corresponding multidimentional mixed integer unbounded knapsack pronlem instance. In this table, $n p$ denotes the problem number. Moreover, TwFP, ToFP and TimFP denote the computing time of the branch and cut method without using the FP, by using the original version of the $F P$ and by using the improved version of the FP, respectively. Finally, $N N w F P, N N o F P$ and $N N i m F P$ denote the number of nodes of the branch and cut tree without using the $\mathrm{FP}$, by using the original version of the $F P$ and by using the improved version of the $\mathrm{FP}$, respectively. In Tables 4 and 5, we compared the number of iterations and the value of the gap resulting from the branch and cut tree for each problem instance. In tables 4 and 5, ITwFP, IToFP and ITimFP denote the number of iterations of the branch and cut method without using the FP, by using the original version of the $F P$ and by using the improved version of the FP, respectively. Moreover, GwFP, GoFP and GimFP denote the gap obtained after the application of the branch and cut method without using the FP, by using the original version of the FP and by using the improved version of the FP, respectively.

The numerical results of tables 2 and 3 show that for 27 instances the best computing times are obtained after the application of the branch and cut method using the original version of the FP. For 30 instances the best computing times are related to the branch and cut method using the improved version of the FP. For 33 problem instances the best computing times are obtained after the application of the branch and cut method without using the FP. Therefore, from 90 test problems, for 57 problem instances using the orignal or the improved version of the $\mathrm{FP}$ reduces the computing time of the branch and cut method.

From 90 generated prolem instances, in 31 cases the branch and cut method without using any versions of the FP has less number of nodes, while in 54 cases using either the original version or improved version of the FP results in less number of nodes of the corresponding branch and cut tree. It can also be verified that for 28 instances using the improved FP results in fewer number of nodes and for 26 instances using the original version gives better results. In 5 cases the number of nodes obtained by using all three branch and cut methods are equal.

In tables 4 and 5, for 31 problem instances the number of iterations of the branch and cut method without using the FP is less than that of the branch and cut method using at least one version of the FP. However, for 57 problem instances the branch and cut method that uses a version of the FP needs less number of iterations. In 34 cases the improved version of the FP needs less number of iterations and in 23 cases the original version of the FP needs less number of iterations. In 2 cases the number of iterations of three branch and cut methods are equal. From the 90 test problems, in 32 test instances, the branch and cut method without using the FP, gives a better value for gap than the branch and cut method using at least a version of the FP, while in 53 cases the branch and cut method using a version of FP gives a better value for the gap. For 31 cases the gap obtained by using the original version of the FP is the best and for 22 instances the improved version of the FP gives the best value for the gap. From the numerical results of tables 1, 2 and 3 we conclude that for most of the test instances, by using the original or improved version of the FP we can improve the efficiency of the resulting branch and cut method. 
Table 1: Properties of the problem instances

\begin{tabular}{cccccccccccc}
\hline$n p$ & $|I|$ & $|C|$ & $|R|$ & $n p$ & $|I|$ & $|C|$ & $|R|$ & $n p$ & $|I|$ & $|C|$ & $|R|$ \\
\hline 1 & 250 & 5 & 50 & 31 & 250 & 20 & 50 & 61 & 500 & 10 & 50 \\
2 & 250 & 5 & 50 & 32 & 250 & 20 & 50 & 62 & 500 & 10 & 50 \\
3 & 250 & 5 & 50 & 33 & 250 & 20 & 50 & 63 & 500 & 10 & 50 \\
4 & 250 & 5 & 50 & 34 & 250 & 20 & 50 & 64 & 500 & 10 & 50 \\
5 & 250 & 5 & 50 & 35 & 250 & 20 & 50 & 65 & 500 & 10 & 50 \\
6 & 250 & 5 & 75 & 36 & 250 & 20 & 75 & 66 & 500 & 10 & 75 \\
7 & 250 & 5 & 75 & 37 & 250 & 20 & 75 & 67 & 500 & 10 & 75 \\
8 & 250 & 5 & 75 & 38 & 250 & 20 & 75 & 68 & 500 & 10 & 75 \\
9 & 250 & 5 & 75 & 39 & 250 & 20 & 75 & 69 & 500 & 10 & 75 \\
10 & 250 & 5 & 75 & 40 & 250 & 20 & 75 & 70 & 500 & 10 & 75 \\
11 & 250 & 5 & 100 & 41 & 250 & 20 & 100 & 71 & 500 & 10 & 100 \\
12 & 250 & 5 & 100 & 42 & 250 & 20 & 100 & 72 & 500 & 10 & 100 \\
13 & 250 & 5 & 100 & 43 & 250 & 20 & 100 & 73 & 500 & 10 & 100 \\
14 & 250 & 5 & 100 & 44 & 250 & 20 & 100 & 74 & 500 & 10 & 100 \\
15 & 250 & 5 & 100 & 45 & 250 & 20 & 100 & 75 & 500 & 10 & 100 \\
16 & 250 & 10 & 50 & 46 & 500 & 5 & 50 & 76 & 500 & 20 & 50 \\
17 & 250 & 10 & 50 & 47 & 500 & 5 & 50 & 77 & 500 & 20 & 50 \\
18 & 250 & 10 & 50 & 48 & 500 & 5 & 50 & 78 & 500 & 20 & 50 \\
19 & 250 & 10 & 50 & 49 & 500 & 5 & 50 & 79 & 500 & 20 & 50 \\
20 & 250 & 10 & 50 & 50 & 500 & 5 & 50 & 80 & 500 & 20 & 50 \\
21 & 250 & 10 & 75 & 51 & 500 & 5 & 75 & 81 & 500 & 20 & 75 \\
22 & 250 & 10 & 75 & 52 & 500 & 5 & 75 & 82 & 500 & 20 & 75 \\
23 & 250 & 10 & 75 & 53 & 500 & 5 & 75 & 83 & 500 & 20 & 75 \\
24 & 250 & 10 & 75 & 54 & 500 & 5 & 75 & 84 & 500 & 20 & 75 \\
25 & 250 & 10 & 75 & 55 & 500 & 5 & 75 & 85 & 500 & 20 & 75 \\
26 & 250 & 10 & 100 & 56 & 500 & 5 & 100 & 86 & 500 & 20 & 100 \\
27 & 250 & 10 & 100 & 57 & 500 & 5 & 100 & 87 & 500 & 20 & 100 \\
28 & 250 & 10 & 100 & 58 & 500 & 5 & 100 & 88 & 500 & 20 & 100 \\
29 & 250 & 10 & 100 & 59 & 500 & 5 & 100 & 89 & 500 & 20 & 100 \\
30 & 250 & 10 & 100 & 60 & 500 & 5 & 100 & 90 & 500 & 20 & 100 \\
\hline & & & & & & & & & & &
\end{tabular}


Table 2: Comparison of the computing time and number of nodes

\begin{tabular}{|c|c|c|c|c|c|c|}
\hline$n p$ & $T w F P$ & ToFP & TimFP & $N N w F P$ & NNoFP & NNimFP \\
\hline 1 & 0.48 & 0.37 & 0.48 & 0 & 496 & 0 \\
\hline 2 & 0.39 & 0.42 & 0.51 & 0 & 0 & 0 \\
\hline 3 & 0.51 & 0.39 & 0.64 & 814 & 1115 & 1772 \\
\hline 4 & 0.38 & 0.36 & 0.36 & 0 & 0 & 0 \\
\hline 5 & 0.38 & 0.33 & 0.36 & 0 & 0 & 0 \\
\hline 6 & 10.4 & 29.45 & 1.61 & 70363 & 240362 & 7456 \\
\hline 7 & 0.61 & 0.72 & 0.61 & 2573 & 4817 & 3104 \\
\hline 8 & 0.61 & 0.86 & 0.69 & 2222 & 4604 & 3426 \\
\hline 9 & 3.21 & 3.01 & 3.50 & 22984 & 22665 & 24414 \\
\hline 10 & 1.56 & 2.64 & 2.42 & 6418 & 17095 & 12683 \\
\hline 11 & 4.71 & 5.10 & 4.58 & 16231 & 22898 & 21290 \\
\hline 12 & 0.75 & 0.83 & 0.95 & 3131 & 2815 & 3877 \\
\hline 13 & 2.08 & 5.72 & 4.18 & 3159 & 20363 & 18535 \\
\hline 14 & 0.55 & 0.78 & 0.55 & 1510 & 4085 & 1565 \\
\hline 15 & 2.22 & 2.34 & 2.48 & 3935 & 5456 & 4996 \\
\hline 16 & 0.41 & 0.37 & 0.44 & 475 & 545 & 0 \\
\hline 17 & 0.50 & 0.47 & 0.45 & 1984 & 1716 & 1495 \\
\hline 18 & 0.80 & 0.76 & 0.83 & 5165 & 4946 & 6138 \\
\hline 19 & 0.47 & 0.47 & 0.55 & 1062 & 1022 & 2545 \\
\hline 20 & 0.41 & 0.42 & 0.36 & 179 & 184 & 170 \\
\hline 21 & 7.68 & 4.57 & 6.72 & 46566 & 22911 & 38360 \\
\hline 22 & 3.79 & 11.23 & 3.74 & 13315 & 49190 & 13496 \\
\hline 23 & 2.22 & 6.33 & 1.50 & 12552 & 30819 & 5542 \\
\hline 24 & 2.84 & 7.58 & 2.95 & 16306 & 29557 & 15507 \\
\hline 25 & 2.17 & 1.26 & 1.43 & 11334 & 4775 & 8146 \\
\hline 26 & 2.14 & 2.03 & 2.06 & 3184 & 3567 & 4767 \\
\hline 27 & 2.62 & 2.59 & 2.71 & 16139 & 3911 & 6764 \\
\hline 28 & 3.09 & 2.40 & 3.96 & 8687 & 8408 & 13681 \\
\hline 29 & 2.93 & 3.23 & 2.54 & 8812 & 7120 & 6903 \\
\hline 30 & 3.21 & 8.74 & 3.03 & 3926 & 36751 & 4010 \\
\hline 31 & 1.34 & 1.79 & 1.84 & 9548 & 14533 & 13736 \\
\hline 32 & 0.81 & 1.03 & 0.81 & 4191 & 5773 & 5528 \\
\hline 33 & 1.50 & 1.17 & 1.18 & 8573 & 5643 & 5353 \\
\hline 34 & 1.08 & 1.15 & 0.65 & 5681 & 5867 & 5048 \\
\hline 35 & 0.45 & 0.50 & 0.50 & 2950 & 2312 & 2469 \\
\hline 36 & 2.79 & 5.16 & 3.26 & 21489 & 33893 & 21768 \\
\hline 37 & 2.00 & 1.67 & 2.21 & 4475 & 4459 & 3777 \\
\hline 38 & 2.34 & 3.20 & 4.31 & 8998 & 16305 & 25723 \\
\hline 39 & 44.24 & 72.62 & 61.01 & 331734 & 632811 & 584904 \\
\hline 40 & 1.67 & 1.75 & 2.36 & 9408 & 8155 & 11937 \\
\hline 41 & 2.70 & 2.82 & 2.84 & 6159 & 5252 & 6584 \\
\hline 42 & 3.20 & 3.99 & 7.36 & 9749 & 15483 & 31502 \\
\hline 43 & 3.65 & 3.99 & 3.85 & 6675 & 6567 & 6743 \\
\hline 44 & 4.85 & 3.01 & 0.51 & 22719 & 8388 & 13400 \\
\hline 45 & 5.29 & 4.15 & 3.84 & 17545 & 8282 & 6160 \\
\hline
\end{tabular}


Table 3: Comparison of the computing time and number of nodes

\begin{tabular}{ccccccc}
\hline$n p$ & TwFP & ToFP & TimFP & NNwFP & NNoFP & NNimFP \\
\hline 46 & 0.76 & 0.69 & 0.69 & 985 & 1700 & 1164 \\
47 & 0.69 & 0.66 & 0.67 & 814 & 812 & 835 \\
48 & 1.03 & 1.16 & 0.89 & 3845 & 3846 & 5811 \\
49 & 0.56 & 0.59 & 0.66 & 1366 & 1662 & 1890 \\
50 & 0.42 & 0.39 & 0.42 & 0 & 0 & 0 \\
51 & 2.39 & 1.93 & 2.04 & 10457 & 9021 & 7551 \\
52 & 12.72 & 4.71 & 2.82 & 67590 & 17050 & 9799 \\
53 & 1.97 & 1.90 & 1.98 & 7612 & 8134 & 6101 \\
54 & 1.83 & 1.67 & 3.38 & 8520 & 2392 & 24692 \\
55 & 2.00 & 1.87 & 1.97 & 6144 & 5765 & 6809 \\
56 & 2.64 & 1.40 & 2.23 & 8598 & 5152 & 5492 \\
57 & 12.71 & 3.59 & 3.12 & 55424 & 14773 & 12746 \\
58 & 1.76 & 1.23 & 1.89 & 2778 & 4219 & 3965 \\
59 & 2.62 & 2.67 & 11.14 & 6089 & 8629 & 46361 \\
60 & 2.81 & 2.40 & & 7968 & 6903 & \\
61 & 2.04 & 2.17 & 2.26 & 5878 & 6068 & 5713 \\
62 & 0.87 & 0.87 & 1.22 & 2684 & 2933 & 4819 \\
63 & 1.23 & 0.86 & 1.29 & 5087 & 4177 & 4718 \\
64 & 1.15 & 1.11 & 1.01 & 4798 & 4469 & 3478 \\
65 & 0.52 & 0.51 & 0.64 & 0 & 0 & 0 \\
66 & 2.34 & 2.03 & 1.86 & 9320 & 6495 & 5602 \\
67 & 7.21 & 13.82 & 13.03 & 25142 & 78036 & 57225 \\
68 & 3.73 & 45.26 & 3.89 & 19411 & 27870 & 18165 \\
69 & 3.34 & 11.33 & 3.25 & 20131 & 48071 & 155487 \\
70 & 2.37 & 3.15 & 2.84 & 8928 & 15889 & 13355 \\
71 & 73.27 & 8.75 & 15.76 & 505691 & 153787 & 323815 \\
72 & 2.01 & 1.67 & 1.26 & 5406 & 4156 & 1627 \\
73 & 2.48 & 2.25 & 2.15 & 3469 & 5717 & 4233 \\
74 & 3.07 & 3.28 & 2.76 & 6565 & 10465 & 6966 \\
75 & 5.02 & 43.79 & 15.12 & 11792 & 272509 & 67032 \\
76 & 1.82 & 2.34 & 5.41 & 1080 & 13394 & 32528 \\
77 & 1.31 & 1.06 & 1.42 & 4536 & 4701 & 6199 \\
78 & 1.34 & 1.20 & 1.47 & 4536 & 5105 & 5189 \\
79 & 1.45 & 1.47 & 1.50 & 5517 & 5655 & 5559 \\
80 & 0.69 & 0.72 & 0.81 & 868 & 884 & 1468 \\
81 & 5.01 & 20.61 & 11.58 & 23232 & 202501 & 56874 \\
82 & 3.24 & 4.94 & 9.22 & 11371 & 21813 & 30544 \\
83 & 188.45 & 489.25 & 17.91 & 1322238 & 2963241 & 127115 \\
84 & 14.54 & 15.59 & 3.32 & 90804 & 88455 & 13174 \\
85 & 3.78 & 14.48 & 4.82 & 9217 & 76941 & 15538 \\
86 & 2.51 & 4.15 & 4.09 & 5940 & 18537 & 16813 \\
87 & 2.29 & 2.22 & 2.23 & 6834 & 5232 & 5223 \\
88 & 2.81 & 2.79 & 1.62 & 4821 & 5043 & 1796 \\
89 & 2.92 & 2.78 & 3.65 & 8293 & 4887 & 4648 \\
90 & 2.03 & 2.14 & 2.07 & 4559 & 5179 & 4445 \\
\hline & & & & & &
\end{tabular}


Table 4: Comparison of the number of iterations and gap

\begin{tabular}{|c|c|c|c|c|c|c|}
\hline$n p$ & $I T w F P$ & IToFP & ITimFP & $G w F P$ & GoFP & GimFP \\
\hline 1 & 750 & 2528 & 793 & 0.25 & - & 3.31622 \\
\hline 2 & 694 & 694 & 694 & - & - & - \\
\hline 3 & 4549 & 4287 & 7769 & 3.039 & 1.329 & 3.010 \\
\hline 4 & 618 & 583 & 591 & 2.475 & - & 0.513 \\
\hline 5 & 722 & 722 & 722 & - & - & - \\
\hline 6 & 485948 & 1726116 & 40445 & - & 4.837 & 4.382 \\
\hline 7 & 12195 & 23514 & 14157 & 4.75 & 4.651 & 4.673 \\
\hline 8 & 17733 & 30941 & 24321 & 3.526 & 3.908 & 2.811 \\
\hline 9 & 22984 & 966356 & 125719 & 4.878 & 5.132 & 4.526 \\
\hline 10 & 33725 & 78151 & 71449 & 4.797 & 5.017 & 4.653 \\
\hline 11 & 130494 & 158240 & 136655 & 6.741 & 6.537 & 6.749 \\
\hline 12 & 13405 & 11719 & 16134 & 6.404 & 6.440 & 6.599 \\
\hline 13 & 17377 & 159901 & 142619 & - & 6.852 & 7.023 \\
\hline 14 & 6446 & 15739 & 5462 & 6.014 & 3.839 & 2.936 \\
\hline 15 & 18820 & 28337 & 30571 & 6.120 & 5.774 & 6.361 \\
\hline 16 & 3132 & 3259 & 813 & 2.041 & 3.107 & - \\
\hline 17 & 8737 & 7192 & 6792 & 1.808 & 1.077 & 0.975 \\
\hline 18 & 23166 & 21720 & 24479 & 2.270 & 1.783 & 3.055 \\
\hline 19 & 5611 & 5575 & 8492 & 2.508 & 2.348 & 3.307 \\
\hline 20 & 1838 & 1882 & 1769 & - & 2.966 & - \\
\hline 21 & 222602 & 137070 & 169458 & 4.810 & 4.824 & 4.841 \\
\hline 22 & 69734 & 391900 & 93071 & 4.704 & 4.921 & 4.930 \\
\hline 23 & 71105 & 177877 & 37672 & 5.118 & 5.056 & 3.258 \\
\hline 24 & 97136 & 220591 & 93723 & 5.037 & 5.102 & 4.343 \\
\hline 25 & 50092 & 25969 & 38112 & 4.972 & 4.466 & 4.858 \\
\hline 26 & 14868 & 16676 & 22994 & 6.317 & 6.797 & 6.793 \\
\hline 27 & 25005 & 18235 & 29694 & 5.343 & 6.304 & 6.197 \\
\hline 28 & 61420 & 52317 & 84621 & 6.446 & 6.813 & 6.705 \\
\hline 29 & 56060 & 34479 & 33405 & 5.415 & 7.105 & 6.696 \\
\hline 30 & 17043 & 193888 & 17431 & 5.681 & 6.820 & 6.539 \\
\hline 31 & 38430 & 60269 & 63205 & 2.403 & 2.682 & 3.240 \\
\hline 32 & 21569 & 21960 & 23102 & 3.129 & - & 3.258 \\
\hline 33 & 27631 & 14572 & 12833 & 3.209 & 3.100 & 3.046 \\
\hline 34 & 22838 & 23210 & 14809 & 3.399 & - & 2.748 \\
\hline 35 & 7774 & 5696 & 5029 & 3.081 & 2.333 & 2.948 \\
\hline 36 & 102539 & 81969 & 81999 & 4.969 & 4.753 & 4.560 \\
\hline 37 & 17051 & 19944 & 13624 & 4.997 & 4.812 & 4.097 \\
\hline 38 & 46620 & 80126 & 143011 & 5.011 & 5.018 & 5.210 \\
\hline 39 & 1697631 & 2953633 & 2149683 & 5.220 & 5.220 & 5.225 \\
\hline 40 & 31955 & 30432 & 62545 & 4.850 & 4.655 & 5.013 \\
\hline 41 & 22306 & 18439 & 25473 & 6.807 & 6.952 & 6.370 \\
\hline 42 & 32451 & 58800 & 112860 & 6.561 & 6.263 & 6.813 \\
\hline 43 & 24258 & 22410 & 21755 & 6.977 & 6.253 & 6.266 \\
\hline 44 & 90375 & 28503 & 47364 & 7.065 & 7.092 & 7.111 \\
\hline 45 & 64412 & 30741 & 23814 & 6.5832 & 6.400 & 6.548 \\
\hline
\end{tabular}


Table 5: Comparison of the number of iterations and gap

\begin{tabular}{|c|c|c|c|c|c|c|}
\hline$n p$ & $I T w F P$ & IToFP & ITimFP & $G w F P$ & GoFP & GimFP \\
\hline 46 & 5816 & 8184 & 6214 & 2.919 & 2.384 & 2.642 \\
\hline 47 & 3963 & 3927 & 3960 & - & - & - \\
\hline 48 & 1483 & 14711 & 23028 & - & 1.747 & 3.097 \\
\hline 49 & 5852 & 5817 & 6508 & 3.158 & 1.537 & 1.976 \\
\hline 50 & 430 & 429 & 407 & - & - & - \\
\hline 51 & 72673 & 46329 & 42139 & 4.888 & 4.769 & 4.739 \\
\hline 52 & 398626 & 148348 & 57436 & 4.889 & 4.815 & 4.555 \\
\hline 53 & 43704 & 40645 & 38372 & 4.204 & 4.750 & 4.821 \\
\hline 54 & 32196 & 27742 & 114320 & 3.688 & 4.113 & 5.157 \\
\hline 55 & 38915 & 33085 & 35581 & 3.330 & 4.762 & 4.693 \\
\hline 56 & 55131 & 44097 & 37867 & 5.759 & 6.151 & 6.489 \\
\hline 57 & 467384 & 88935 & 84622 & 6.563 & 5.737 & 6.573 \\
\hline 58 & 19529 & 30550 & 26879 & - & 5.963 & 2.030 \\
\hline 59 & 37525 & 54120 & 378039 & 5.484 & 6.867 & 7.058 \\
\hline 60 & 53390 & 53275 & & 6.096 & 6.540 & \\
\hline 61 & 25099 & 24664 & 22851 & 0.873 & 1.381 & 0.418 \\
\hline 62 & 12205 & 14301 & 23259 & 2.193 & 2.749 & 2.257 \\
\hline 63 & 18579 & 14928 & 14469 & 3.025 & 2.572 & 0.203 \\
\hline 64 & 20051 & 18348 & 11369 & 3.059 & 1.657 & 3.23 \\
\hline 65 & 683 & 736 & 846 & 1.766 & 2.739 & - \\
\hline 66 & 60657 & 36264 & 30635 & 3.857 & 4.019 & 4.672 \\
\hline 67 & 212605 & 433766 & 476890 & 4.808 & 4.942 & 4.917 \\
\hline 68 & 90872 & 1910354 & 95628 & 4.992 & 5.103 & 5.007 \\
\hline 69 & 117520 & 352917 & 87444 & 5.183 & 5.197 & 4.952 \\
\hline 70 & 38216 & 70399 & 55249 & 5.026 & 4.995 & 4.838 \\
\hline 71 & 2987870 & 153787 & 323819 & 6.926 & 6.919 & 6.919 \\
\hline 72 & 67537 & 38813 & 16345 & 6.826 & 6.018 & 4.230 \\
\hline 73 & 20075 & 36243 & 21245 & 2.263 & 5.776 & 4.592 \\
\hline 74 & 47292 & 91815 & 46664 & 7.003 & 6.664 & 6.862 \\
\hline 75 & 56764 & 1562728 & 474330 & 6.611 & 6.777 & 6.767 \\
\hline 76 & 36240 & 48065 & 146609 & 2.770 & 3.291 & 3.193 \\
\hline 77 & 15961 & 16799 & 25469 & - & 2.402 & 3.191 \\
\hline 78 & 15961 & 19127 & 17103 & - & 2.817 & 2.274 \\
\hline 79 & 17230 & 21117 & 21501 & 0.934 & 3.190 & 3.164 \\
\hline 80 & 3190 & 3382 & 6151 & - & 1.224 & 3.320 \\
\hline 81 & 87545 & 833289 & 286222 & 4.895 & 4.902 & 4.869 \\
\hline 82 & 62544 & 143951 & 225584 & 5.030 & 5.017 & 5.012 \\
\hline 83 & 10028928 & 24535349 & 609941 & 5.115 & 5.115 & 5.112 \\
\hline 84 & 406629 & 487306 & 46243 & 5.251 & 5.268 & 4.978 \\
\hline 85 & 34029 & 458821 & 71820 & 5.069 & 5.100 & 4.972 \\
\hline 86 & 22881 & 82056 & 84012 & 6.678 & 6.978 & 6.821 \\
\hline 87 & 24985 & 19732 & 20073 & 6.831 & 6.618 & 6.490 \\
\hline 88 & 16404 & 19021 & 6976 & 5.801 & 6.034 & 6.953 \\
\hline 89 & 32017 & 20043 & 19814 & 6.942 & 7.187 & 6.472 \\
\hline 90 & 18980 & 22093 & 18513 & 4.493 & 6.431 & 5.468 \\
\hline
\end{tabular}




\section{Acknowledgements}

The authors thank the Shiraz university of Technology for supporting this work.

\section{References}

[1] A. Atamturk, On the facets of the mixedinteger knapsack polyhedron, Mathematical Programming Serries B, 98: 145175, 2003.

[2] Boland N. L., Eberhard A.C., Engineer F. and Tsoukalas A, Improving the feasibility pump. Discrete Optimization, 4(1):77-86, 2007.

[3] Bertacco L. Fischetti M. and Lodi A, A feasibility pump heuristic for general Mixed-Integer Problems. Discrete Optimization, 4(1):63-76, 2007.

[4] M. Elf, C. Gutwenger, M. Junger and G. Rinaldi, branch and cut Algorithm for combinatorial optimization and their implementation in ABACUS,Computational Combinatorial Optimization: Optimal or Provably Near-Optimal Solutions., Lecture Notes in Computer Science. 2241 Springer 2001, pp. 157-222.

[5] H. Kellerer, U. Pferschy and D. Pisinger, Knapsack Problems, Springer-Verlag, Berlin, 2004.

[6] R. Weismantel, On the 0/1 knapsack polytope. Mathematical Programming, 77:4968, 1997.

[7] L. A. Wolsey, Integer Programming, John Wiley and Sons, New York, 1998. 